\title{
Link Between SCN5A Mutation and the Brugada Syndrome ECG Phenotype Simulation Study
}

\author{
Shunichiro Miyoshi, MD*; Hideo Mitamura, MD*; Yukiko Fukuda, MD; \\ Kojiro Tanimoto, MD; Yoko Hagiwara, MD; Hideaki Kanki, MD; \\ Seiji Takatsuki, MD; Mitsushige Murata, MD; \\ Toshihisa Miyazaki, MD; Satoshi Ogawa, MD
}

\begin{abstract}
Background The specific changes in the gating kinetics of the sodium current ( $\mathrm{INa}_{\mathrm{Na}}$ responsible for its phenotype have remained to be elucidated. In the present study the effect of changes in the gating kinetics of INa on early repolarization (ER) and initiation of phase 2 reentry (P2R) were evaluated in a theoretical epicardial ventricular fiber model.

Methods and Results Miyoshi-ICaL was incorporated into the modified Luo-Rudy dynamic (LRd) model. Dispersion at Ito-density was set within a theoretical fiber composed of serially arranged epicardial cells with gap junctions. The following changes in INa kinetics were made: (1) a $-10 \mathrm{mV}$ shift in steady-state inactivation, (2) a $+10 \mathrm{mV}$ shift in steady-state activation curve, (3) a small inactivation time constant (DEC); P2R and ER were observed. A conduction disturbance within the fiber was simulated and only when the INa-density was decreased did DEC, especially, show a marked increase in the likelihood of causing ER and P2R. Conduction disturbance significantly increased the likelihood causing ER or P2R.

Conclusions In this one-dimension model with Ito-density dispersion, DEC-INa precipitates INa-blocker inducible ER. This suggests that the characteristic ST-segment elevation in the Brugada syndrome with SCN5A mutation can be interpreted in part by DEC-INa. Concomitant conduction disturbance may be required to cause P2R at physiological Ito density. (Circ J 2005; 69: 567-575)
\end{abstract}

Key Words: Arrhythmia; Computers; Electrocardiogram; Electrophysiology; Ventricles

B rugada syndrome (BS) is characterized by ST-segment elevation in the right precordial lead and sudden cardiac death in the absence of obvious organic heart disease! The temporal correlation between increased ST-segment elevation and the occurrence of ventricular fibrillation (VF) originating in the right ventricle (RV) suggests that the mechanism of the ST-segment elevation must be the key to the initiation of VF in BS?,3 Since Ito is prominent in the RV epicardium, ${ }^{4}$ Ito-mediated early repolarization (ER) of the action potential can be observed preferentially in RV epicardium, leading to STsegment elevation via an increasing transmural gradient of the membrane potential3,5 This is thought to be one of the possible cellular mechanisms of the ST-segment elevation in BS, $3,5,6$ The transmural dispersion of repolarization in the $\mathrm{RV}$ is capable of precipitating phase 2 reentry (P2R) and subsequent circus movement reentry, $5,7-9$ but because it does not always do so, the precise mechanisms of P2R and VF in BS have remained unclear.

The finding of enhanced ST-segment elevation by fast sodium current ( $\mathrm{INa}$ ) blockers ${ }^{3,10}$ and of the SCN5A-muta-

(Received September 24, 2004; revised manuscript received February 7, 2005; accepted February 15, 2005)

Cardiopulmonary Division, *Advanced Cardiac Therapeutics, Keio University School of Medicine, Tokyo, Japan

Mailing address: Shunichiro Miyoshi, MD, PhD, Cardiopulmonary Division of Keio University School of Medicine, 35-Shinanomachi, Shinjuku-ku, Tokyo 160-8582, Japan. E-mail: smiyoshi@cpnet.med. keio.ac.jp tions in $\mathrm{BS}^{11-16}$ suggests that INa as well as Ito plays a major role in the pathogenesis of BS. Although various abnormalities in the current phenotype have been reported in SCN5A mutations, it is difficult to identify the abnormality in kinetics that causes specific phenotypes of the disease in experimental models. Various computer simulations have shown that the current phenotype of the mutant currents can cause ER, but they failed to simulate $\mathrm{P} 2 \mathrm{R},{ }^{6,12,17}$ and thus the insight for the arrhythmogenic mechanism in BS was limited.

The marked cell-to-cell variance in Ito-density within the $\mathrm{RV}$-epicardium can generate significant dispersion in action potential duration, which has been thought to cause P2R within the RV4 Therefore previously, we composed new 1-dimensional epicardial fiber model in which we set the dispersion in Ito-density to simulate $\mathrm{P} 2 \mathrm{R} !^{18}$ In the previous model, we re-measured the voltage dependent kinetics of the L-type Ca current (ICaL) precisely and then composed new mathematical model of ICaL that was essential to simulate P2R. In the present study we evaluated how such kinetic changes affect the occurrence of P2R and ER in our model by modifying the voltage-dependent kinetics of INa according to observations that have been reported in the Brugada-related SCN5A mutations!1-16 We also investigated the effect of INa blockade in each simulated SCN5A mutation to identify the mechanism of the INa-blockerinduced ST-segment elevation only in patients with BS3,10

On the other hand, because prolongation of the His-ventricular (HV) interval ${ }^{1,3}$ and a delayed potential detected in 


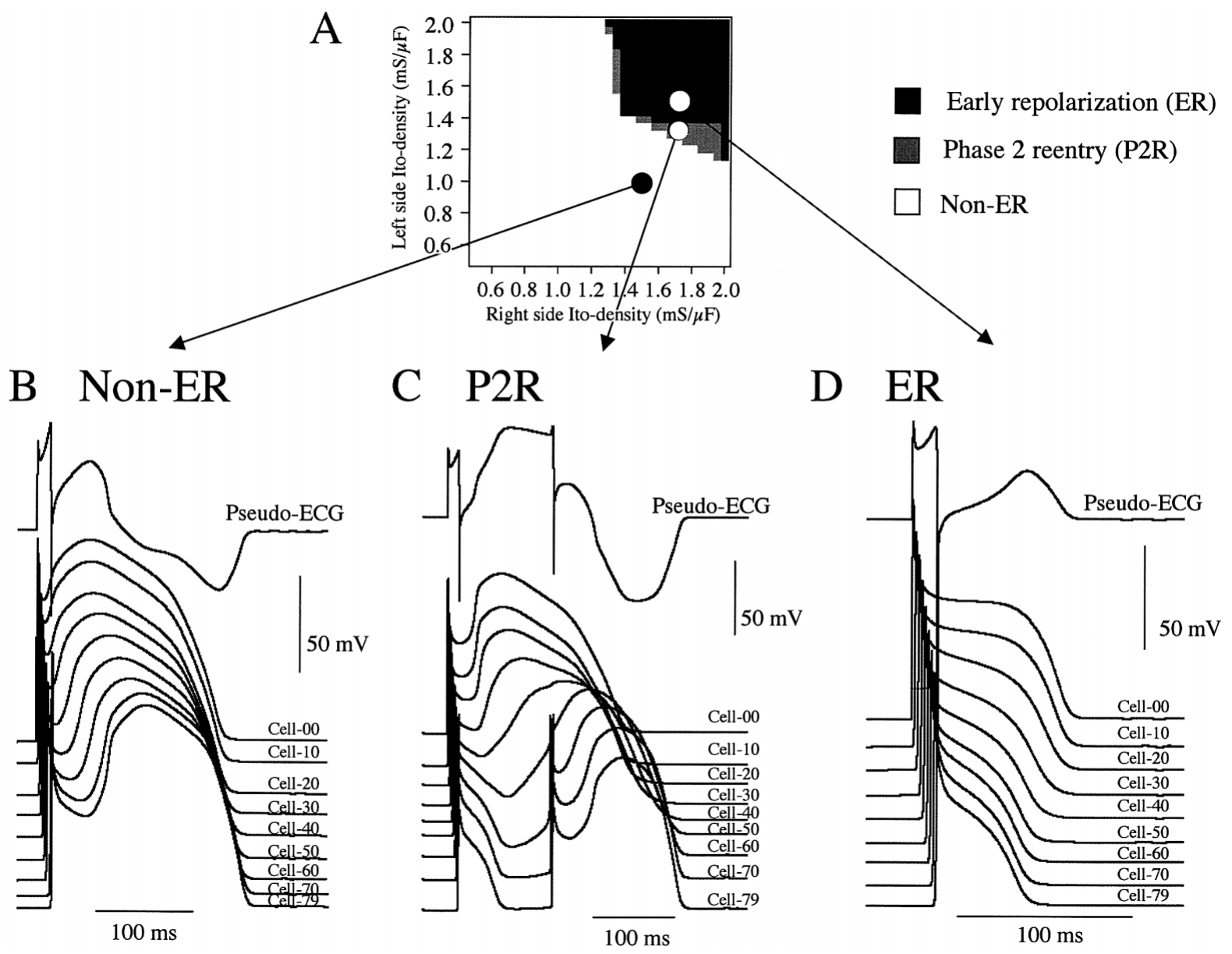

Fig 1. Occurrence of P2R and ER by dispersion at an Ito-density. (A) The vertical axis represents the Ito-density at the cells on the left side of the theoretical epicardial fiber, and the horizontal axis represents the right side. Each circle symbol represents a pixel from which 3 representative sets of traces (B, C, D) were obtained. See text for details. Please note that the time scale of panel D is longer than for B \& C.

the signal-averaged electrocardiogram (ECG) $)^{3,19,20}$ are commonly observed in BS, the presence of diseased myocardium with a conduction disturbance has been thought to be a cellular basis for the syndrome ${ }^{21}$ Isolated RV myocytes are usually smaller in diameter than isolated left ventricular myocytes 22,23 and because smaller cell diameter increases intracellular resistance along the longitudinal axis, the conduction disturbance may be caused preferentially in the $\mathrm{RV}$. Therefore, in the present study we simulated a conduction disturbance by changing the cell diameter in order to observe the occurrence of P2R and ER in the model.

\section{Methods}

\section{Epicardial Fiber Model}

The epicardial fiber model previously described was used 18 The mathematically defined model of Ito devised by Dumaine ${ }^{12}$ and our own model of $\mathrm{ICaL}^{18}$ were incorporated into the Luo-Rudy dynamic (LRd) model ${ }^{24}$ to simulate epicardial action potentials. The theoretical fiber ${ }^{25}$ was composed of 50-150 serially arranged epicardial ventricular cells (total fiber length $0.5-1.5 \mathrm{~cm}$ ) with gap junctions. No flux boundary conditions were used at either end of the fibers. The intracellular resistance per element unit $(\mathrm{Ri})$ is composed of myoplasmic resistance $\left(R_{m y o}=150 \Omega \mathrm{cm}\right)$ and gap junction resistance $\left(\mathrm{Rg}=1.5 \Omega \mathrm{cm}^{2}\right)^{25} \Delta \mathrm{x}=100 \mu \mathrm{m}$ (entire cell length) is used as the discretization element, with $\mathrm{R}_{\mathrm{i}}$ reflecting the lumped contribution of axial and gap junction resistance $(\mathrm{Ri}=\mathrm{R} \text { myo }+\mathrm{Rg} / \Delta \mathrm{x})^{25}$ Differential equations were solved numerically by using the CrankNicholson time derivative ${ }^{26}$ set at $10 \mu \mathrm{s}$, and the space derivative was set at $0.1 \mathrm{~mm}$. Maximal ICaL-conductance was increased $20 \%$ over the original Miyoshi-ICaL ${ }^{18}$ value in every experiment for the following reasons. In the previous study, we set the same maximum conductance of the ICaL as in the Dumaine's model in order to compare the Rasmusson's-ICaL model, but the phase 1 magnitude was quite low $(<-30 \mathrm{mV})$ compared with the experimental data 4 In the previous study, the occurrence of $\mathrm{P} 2 \mathrm{R}$ was too low to observe the change in the occurrence of $\mathrm{P} 2 \mathrm{R}$ induced by the INa mutation, so empirically we set the ICaL conductance as $120 \%$ of our previous paper. We set the dispersion at the Ito-density at the both end of the fiber and applied pacing to the cell at the left end so as to generate P2R in the fiber. In order to excite the cell the current was transmembranously injected into the cell with $0.5 \mathrm{~ms}$ duration and $2 \times$ threshold intensity. We observed the action potential propagation pattern elicited by the first pacing. We defined a P2R impulse that traveled from left to right as an antidromic P2R, and a P2R from right to left as an orthodromic P2R. As shown previously 18 it was difficult to distinguish an antidromic P2R from an electrotonic reflection?27,28 However, 


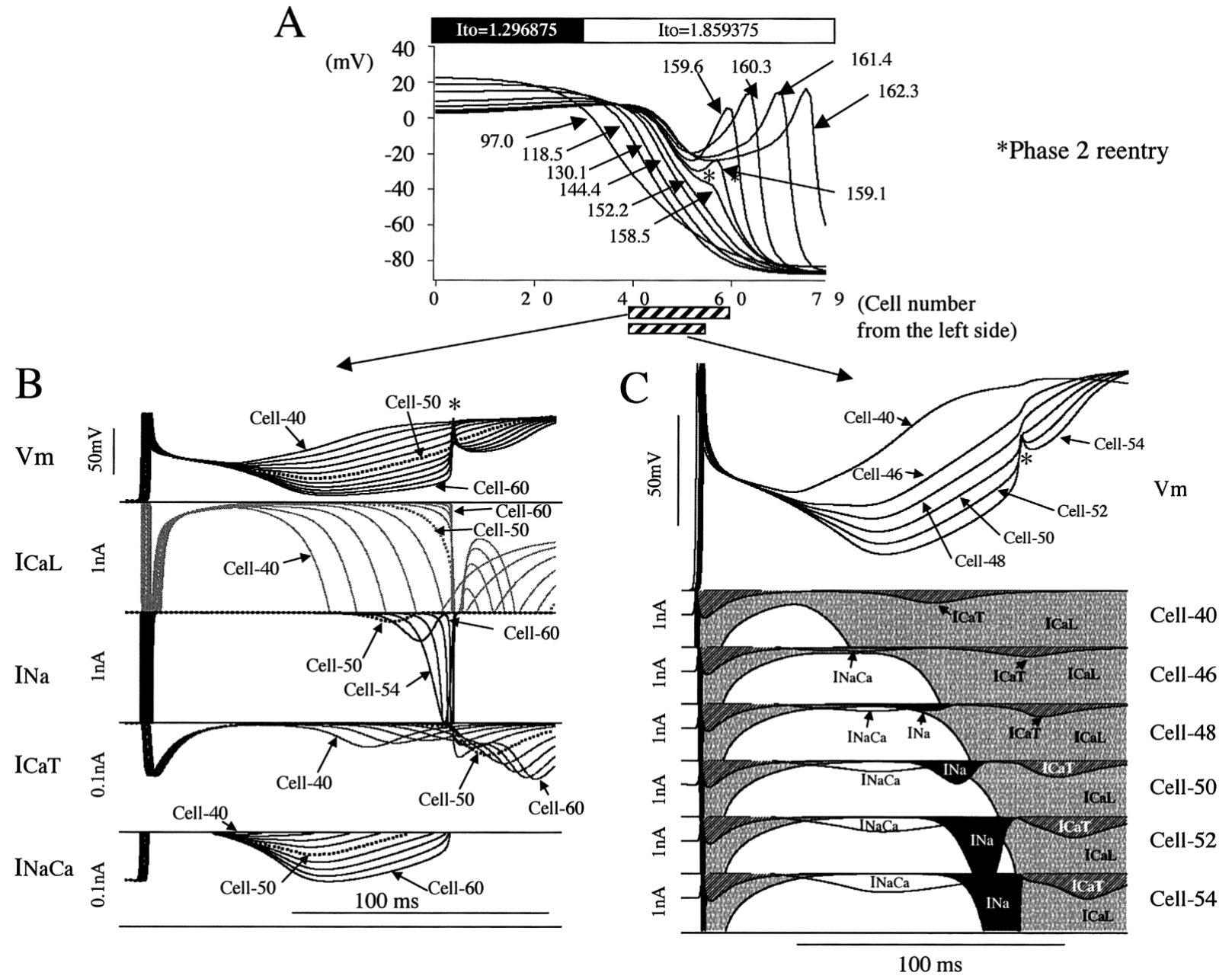

Fig 2. Representative phase 2 reentry trace in the mathematical model and the role of inward currents. (A) Membrane potentials in the fiber at each point in time are shown. The horizontal axis indicates the distance of the cell from the left end of the fiber, and the vertical axis indicates the membrane potential. Each line represents the membrane potential at each point after the start of pacing (denoted along beside). The top bar represents the distribution of cells with each Itodensity shown within the bar. The phase 2 dome was conducted from left to right and generated another action potential (*). The striped bars at the bottom represent the range of cells, and their membrane potentials, and the inward currents were precisely observed and are shown in the lower panels. (B) Calculated currents and potentials of each numbered cell are superimposed on each horizontal column. The upper limit in the current column is zero, and the full scale is shown to the left of each column. (C) The currents of the numbered cells (denote the right) are superimposed on each column. The vertical axis is the same as in (B). INaCa, INa, ICaT, and ICaL are shown in white, black, dark gray, and light gray, respectively. A peak INa that was larger than the ICaL and preceded the second action potential of phase 2 reentry $(*)$ was observed in Cells 50-54.

as no antidromic P2R was observed at the end of the fiber, the mechanism of the antidromic P2R differed from that of electrotonic reflection at the dead end of the fiber. Because we preferred to observe the orthodromic P2R, which is usually referred to as $\mathrm{P}_{2} \mathrm{R}^{27}$ we changed the Ito-density in the $63 \%$ cells on the right side of the fiber from that of the $37 \%$ cells on the left side to generate the dispersion to observe $\mathrm{R} 2 \mathrm{R}$ in the fiber on the right side precisely. The Itodensities were raised from 0.5 to $2 \mathrm{mS} \mu \mathrm{F}$ in increments of 0.09375, and the fiber was observed for the occurrence of $\mathrm{P} 2 \mathrm{R}$ and ER. The physiological Ito range in the RV epicardium was set at less than $1.1 \mathrm{mS} \mu \mathrm{F}$ in the present study $12 \mathrm{~A}$ $\mathrm{P} 2 \mathrm{R}$ was defined as 2 action potentials separated by full repolarization of less than $-80 \mathrm{mV}$ generated by a single ventricular pacing. Because the currents through the cell membrane at both dead ends of the fiber are necessarily over-estimated, the P2R data generated from the last 3 cells at each end of the fiber were discarded. To simulate conduction disturbances, we modified the $\mathrm{Rg}$ and monitored the fiber for the occurrence of ER and P2R. The conduction velocity $(\mathrm{CV})$ was measured by the impulse propagation speed between the cell-30 to cell-50 (m/s). A pseudoECG $^{29}$ was calculated as an extracellular field potential along the axis of our theoretical fiber and $4 \mathrm{~cm}$ from the right end of the fiber.

\section{Change in Sodium Current Kinetics}

Various mutations in the SCN5A channel have been reported ${ }^{11-16}$ in BS. In the present study, we simulated several types of mutations that can decrease INa conductance. We set the INa mutation as follow: (1) a $-10 \mathrm{mV}$ shift in steadystate inactivation curve (InACT) $13,14,16$ (2) a $+10 \mathrm{mV}$ shift in steady-state activation curve (ACT), ${ }^{13,16}$ and (3) a small inactivation time constant (half of the original LRd) 
A pilsicainide $150 \mathrm{mg} / \mathrm{po}$

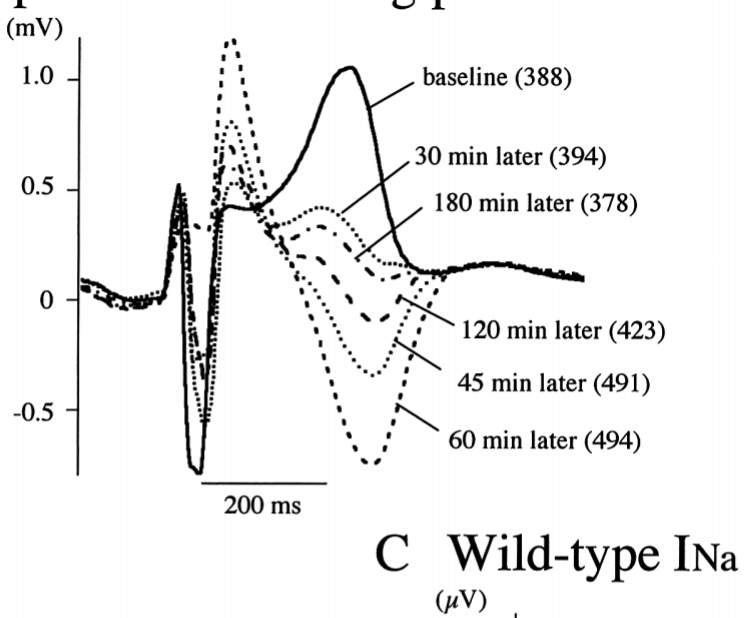

B DEC-INa

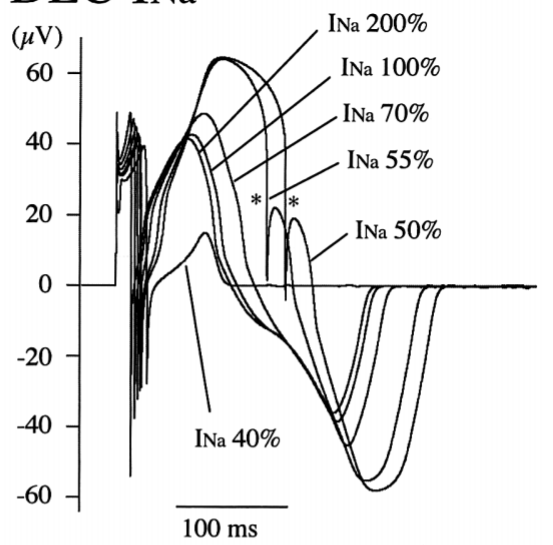

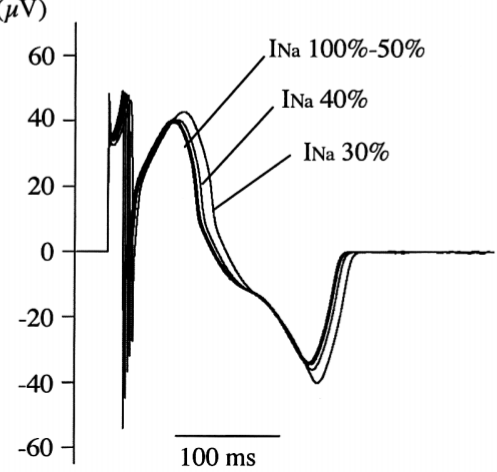

Fig 3. Effect of an INa blocker on the ECG. (A) Superimposed representative ECG ( $\left.\mathrm{V}_{2}\right)$ tracings from a patient with Brugada syndrome before and after pilsicainide administration. Time after pilsicainide and the calculated QTc interval are shown beside each trace. The effect of a change in INa-density on the extracellular field potential (Pseudo-ECG) calculated from the theoretical epicardial fiber with DEC-INa (B) and with wild-type INa (C) are shown. INa-density was varied between $30 \%$ to $100 \%$ (noted beside the trace) of the original LRd model.

(DEC) ${ }^{12,16}$ We incorporated the simulated SCN5A mutations in our epicardial model, and then monitored the fiber for the occurrence of ER and P2R.

\section{ECG From a Patient With BS}

An ECG was obtained from a patient with BS during a pilsicainide test (150 mg po). The patient was a 45 -year-old Japanese male with a family history of sudden death at 41 years of age. The patient gave informed consent for the challenge test, and the institutional review board approved the protocol. The mutations of ion channels in the patient are unknown, because he did not agree to genetic analysis.

\section{Results}

The spatial dispersion in Ito density within the theoretical fiber $(0.8 \mathrm{~cm})$ sometimes generated P2R and ER, and sometimes did not (Non-ER). The relationship between the P2R and the Ito densities in the fiber is shown in the matrix sheet (Fig 1A). The data are symbolized as colored pixels on a coordinate plane the vertical axis of which represents Ito density in the fiber on the left side (Left fiber) and the horizontal axis represents Ito density in the fiber on the right side (Right fiber). In Fig 1B, the right cell showed accentuation of the phase 1 notch and a slight increase in the action potential duration; however, no cell in the fiber showed ER or P2R. The pseudo-ECG showed mild accentuation of the R'-wave (J-wave). A small inverted T-wave was observed, but it may have been masked by the large Twave of LV origin because the action potential duration is longer in the LV than in the RV. The pseudo-ECG showed a coved-type ST-segment elevation when P2R occurred in the fiber (Fig 1C). When ER occurred (Fig 1D) there was marked shortening of the QT interval. The present model is an epicardial 1-D fiber model, so there is no transmural axis (along the axis from endo-epi). Gima and Rudy reported that epicardial ER was observed as a saddle-back type STsegment elevation on the ECG in a transmural 1-D fiber model6

To evaluate the likelihood of arrhythmogenesis, we calculated the pixel area of P2R or ER $+\mathrm{P} 2 \mathrm{R}$. It is known that Ito varies beat to beat as a function of the preceding diastolic interval, so we assumed if the pixel area was large, P2R would occur more easily by chance. Furthermore only the $\mathrm{P} 2 \mathrm{R}$ observed in the physiological range is significant. Because P2R usually occurs at the margin between the area of ER and non-ER, the large net pixel area of ER and P2R corresponds to the occurrence of $\mathrm{P} 2 \mathrm{R}$ at the low end close to physiological Ito density. Therefore it is important to note that both the large net pixel area of ER $+\mathrm{P} 2 \mathrm{R}$ and a large pixel area of $P 2 R$ are necessary to generate $P 2 R$ in $\mathrm{BS}$. Furthermore, the net pixel area of ER and P2R also corresponds to the probability of causing a saddle-back type and coved-type ST-segment elevation, respectively.

In the latter part of the present study, we calculated the pixel area in the matrix data. A representative P2R in the 


\section{Wild-type INa}

INa $100 \%$

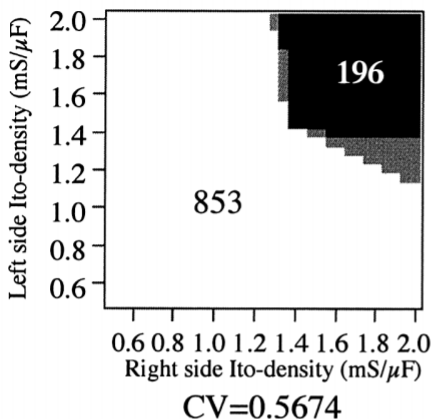

DEC INa

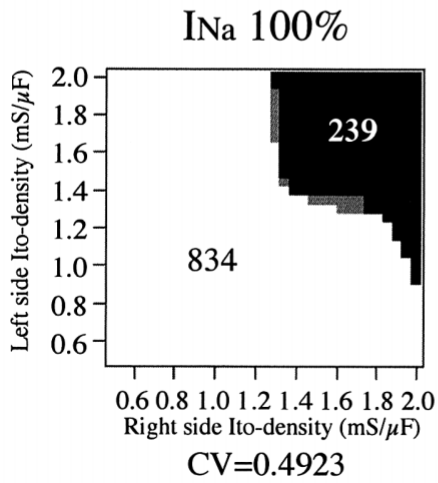

INa $70 \%$

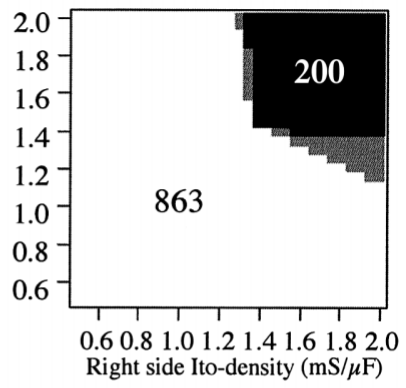

$\mathrm{CV}=0.5031$
INa $50 \%$

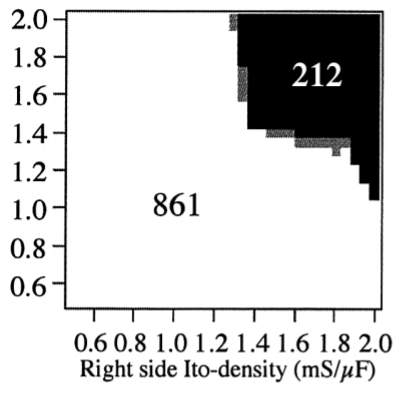

$\mathrm{CV}=0.4457$
INa $40 \%$
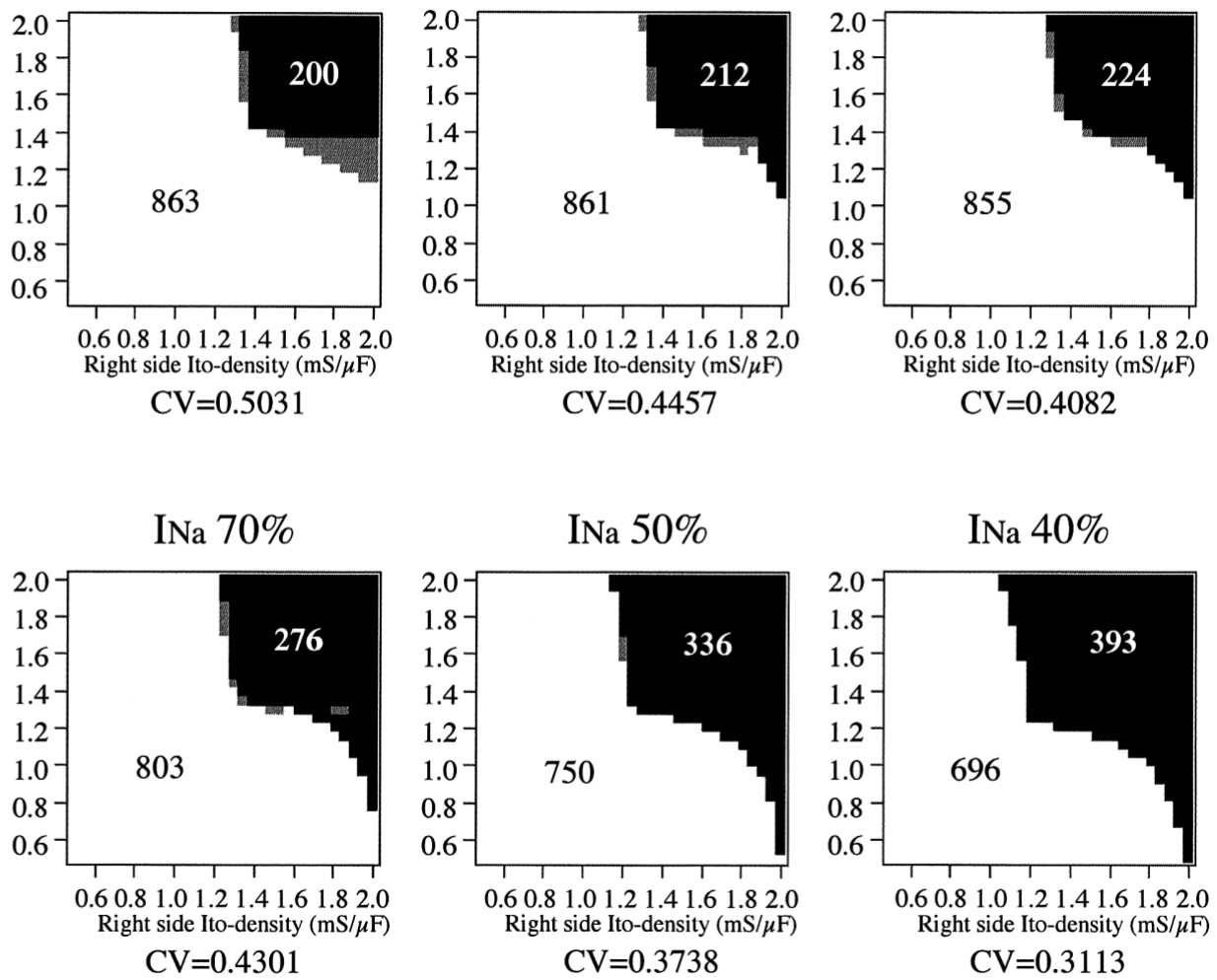

$\mathrm{CV}=0.4301$

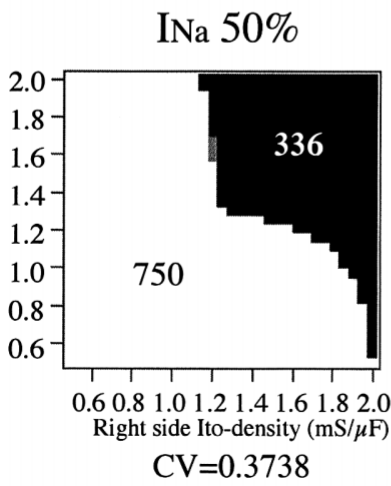

Phase 2 reentry (P2R)
INa $40 \%$

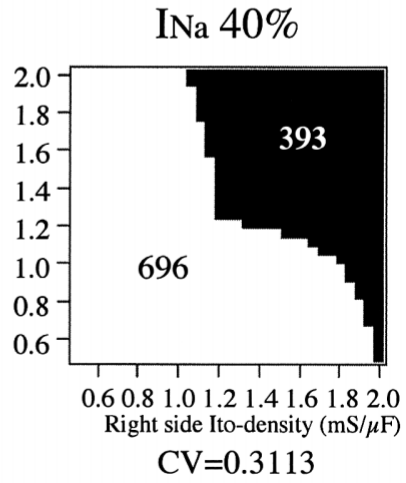

Non-ER

Fig 4. Occurrence of P2R and ER as a result of dispersion at an Ito-density with wild-type INa (Upper panels) or DEC-INa (Lower panels) at various Ina-densities are shown. Each matrix is shown in the same manner as in Fig 1. Ina density is shown at the top of each matrix, and the calculated CV is shown at the bottom of each matrix. The numbers in the matrix are the numbers of white and black pixels. Decreases in INa density did not increase the area of P2R and ER greatly in the matrix with the wild-type INa, but they increased the pixel area dramatically in the matrix with DEC-INa. CV, conduction velocity.

fiber $(0.8 \mathrm{~cm})$ is shown in Fig $2 \mathrm{~A}$. The action potential at the right fiber has lost its dome and has repolarized. The phase 2 dome in the left fiber gradually propagated to the right, and finally generated a P2R (*) in an "all or none" manner. The "all or none" excitation of $\mathrm{P} 2 \mathrm{R}$ propagated to the right side of the fiber. The ICaL, INa, T-type Ca current (ICaT), and the $\mathrm{NaCa}$ exchanger current $(\mathrm{INaCa})$ at the time of the onset of P2R are shown in Fig 2B,C. The major inward current in the cells to the left of Cell 50 was ICaL, which passed into the cell on the right fiber via a gap junction and caused slow depolarization. As discussed in a previous paper, ${ }^{18}$ this current is mainly caused by reactivation after deactivation of ICaL, which is described as the "d-gate" in the mathematical model. However, the P2R did not always occur in an "all or none" manner only by this reactivation of ICaL. Both INaCa and INa may play a role in this slow depolarization; however, because INaCa itself does not cause self-reproducing depolarization, it must not play a major role in triggering P2R. It is noteworthy that in Cells 50-54, the peak INa, which was greater than the ICaL, preceded the initiation of P2R (*) and ICaL activation, suggesting that the INa must play an important role in the "all or none" activation of the second action potential of the P2R.

A representative ECG trace during the pilsicainide test in the patient with $\mathrm{BS}$ is shown in Fig $3 \mathrm{~A}$. No genetic data were available for his ion channel mutation. The V2-lead tracings before and after pilsicainide administration have been digitized and superimposed. Pilsicainide unmasked the coved-type ST-segment elevation, whereas the baseline ECG showed a saddleback-type ST-segment elevation. The amplitudes of the R'-wave and negative T-wave increased as a function of time after administration of pilsicainide. At $60 \mathrm{~min}$, when the response to pilsicainide was maximal, QTc was markedly prolonged and the isoelectrical line of the ST-segment disappeared. In the simulation with DECINa, INa blockade had a similar effect on the pseudo-ECG (Fig 3B). Increases in amplitude of the R'-wave and negative T-wave and QT-prolongation were observed as clear functions of decreasing INa-density. If the P2R was blocked within the local RV epicardium, we would not observe it as PVC, but might observe it as a coved-type ST-segment elevation in the right precordial leads. Blockade of INa, on the other hand, had little effect on the pseudo-ECG in the simulation with wild-type INa.

The effect of mutation in INa on ER and P2R is shown in Fig $4(0.8 \mathrm{~cm})$. DEC-INa decreased the conduction velocity and the pixel area of P2R, but the net pixel area of P2R and ER did not change much. When INa-density was decreased in the simulation with DEC-INa, however, the net pixel area of P2R and ER increased dramatically, and there was only 
Area of ER+P2R

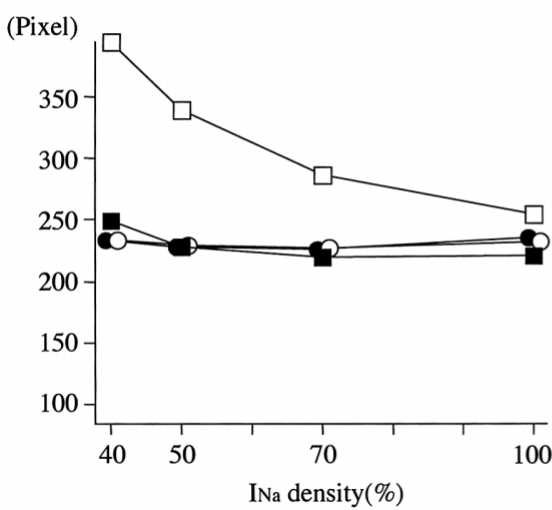

Area of P2R

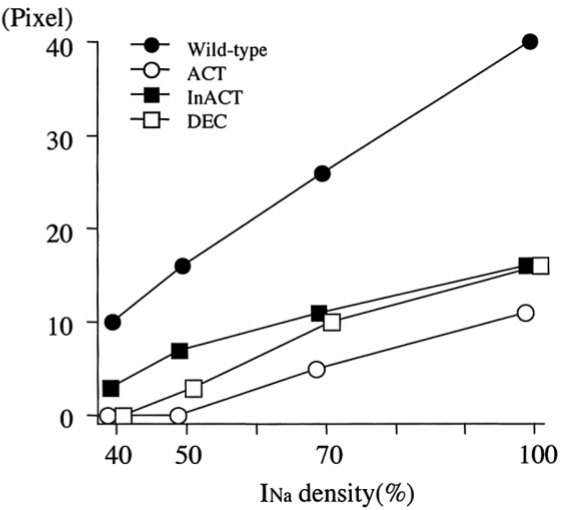

Fig 5. Effect of reducing INa density on the calculated pixel area of ER +P2R (Left panel) and P2R (Right panel) in the presence of various simulated INa mutations. At an INa density of $100 \%$, the ER+P2R pixel area did not change very much. However, $\mathrm{ER}+\mathrm{P} 2 \mathrm{R}$ increased dramatically as a function of decreases in INa-density only in DEC-INa, and not with the other simulated wild-type and mutant INa. The area of P2R seems to decrease as a function of decreases in INa-density. ER, early repolarization; P2R, phase 2 reentry.

\section{Early repolarization (ER) \\ Phase 2 reentry (P2R) \\ Non-ER}
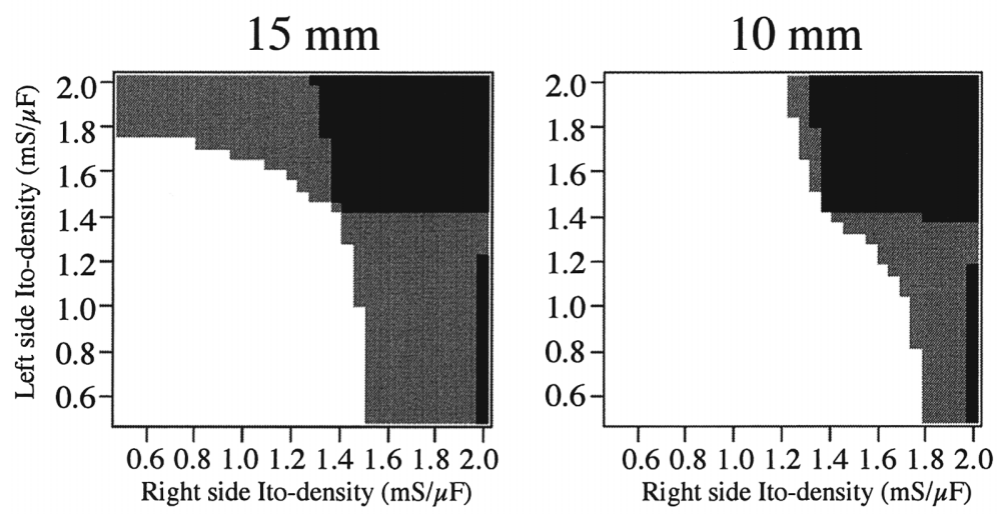

\section{$8 \mathrm{~mm}$}

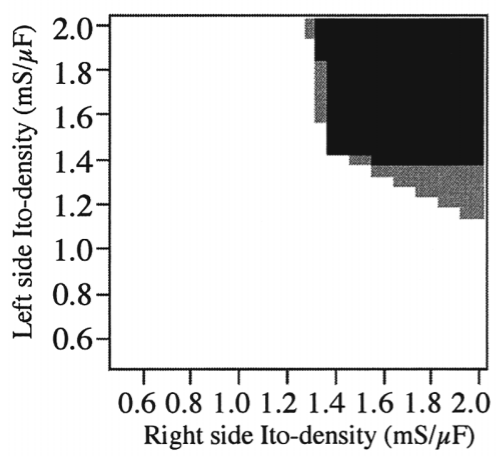

\section{$5 \mathrm{~mm}$}

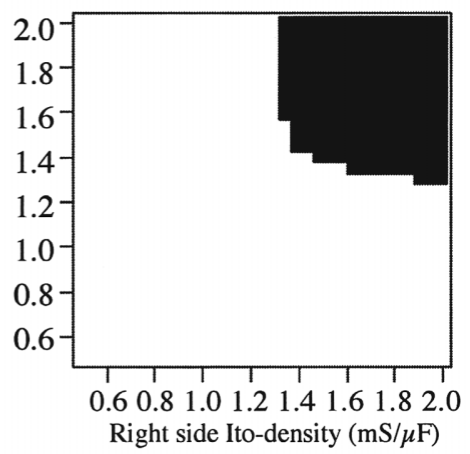

Fig 6. Occurrence of P2R and ER as a result of dispersion at an Ito-density with wild-type INa in various lengths of epicardial fiber. Each matrix is shown in the same manner as in Fig 1. Fiber length is shown at the top of each matrix. The pixel area of P2R increased dramatically as a function of increases in fiber length, but ER did not change very much.

a small change in the wild-type INa. Interestingly, the pixel area of the P2R was decreased by the INa-reduction. The pixel areas of ER and P2R calculated for various simulated SCN5A mutations and Ina-densities are shown in Fig 5. Neither ACT nor InACT increased the ER pixel area very much compared with the wild-type INa. Although DEC-INa increased the ER pixel area only slightly at normal INadensity, it increased dramatically at the lower INa-density.

We then investigated the effect of changes in the total length of the fiber on the ER and P2R (Fig6). The pixel area of P2R increased markedly as a function of increases in total fiber length. Conduction disturbance also plays an important role in the occurrence of P2R (Fig 7) in the
$0.8 \mathrm{~cm}$ epicardial fiber. The pixel areas of P2R and ER in the matrix increased as a function of decreases in the conduction velocity induced by changing the radius of the cell. At the baseline INa-density, the net pixel areas of ER and P2R were similar in the simulation with both wild-type INa and DEC-INa, whereas, at decreased INa-density, the pixel areas of ER and P2R were markedly increased only when simulated with DEC-INa, especially in the smaller diameter cells, and thus the P2R occurred much closer to the physiological Ito-density (dotted box) in the simulation with a cell radius of $5 \mu \mathrm{m}$ and a DEC-INa. Despite the conduction velocity being slower in the simulation with wild-type INa, $\mathrm{r}=5 \mu \mathrm{m}$, and $40 \%$ INa density than it with DEC-INa, $\mathrm{r}=9 \mu \mathrm{m}$, 

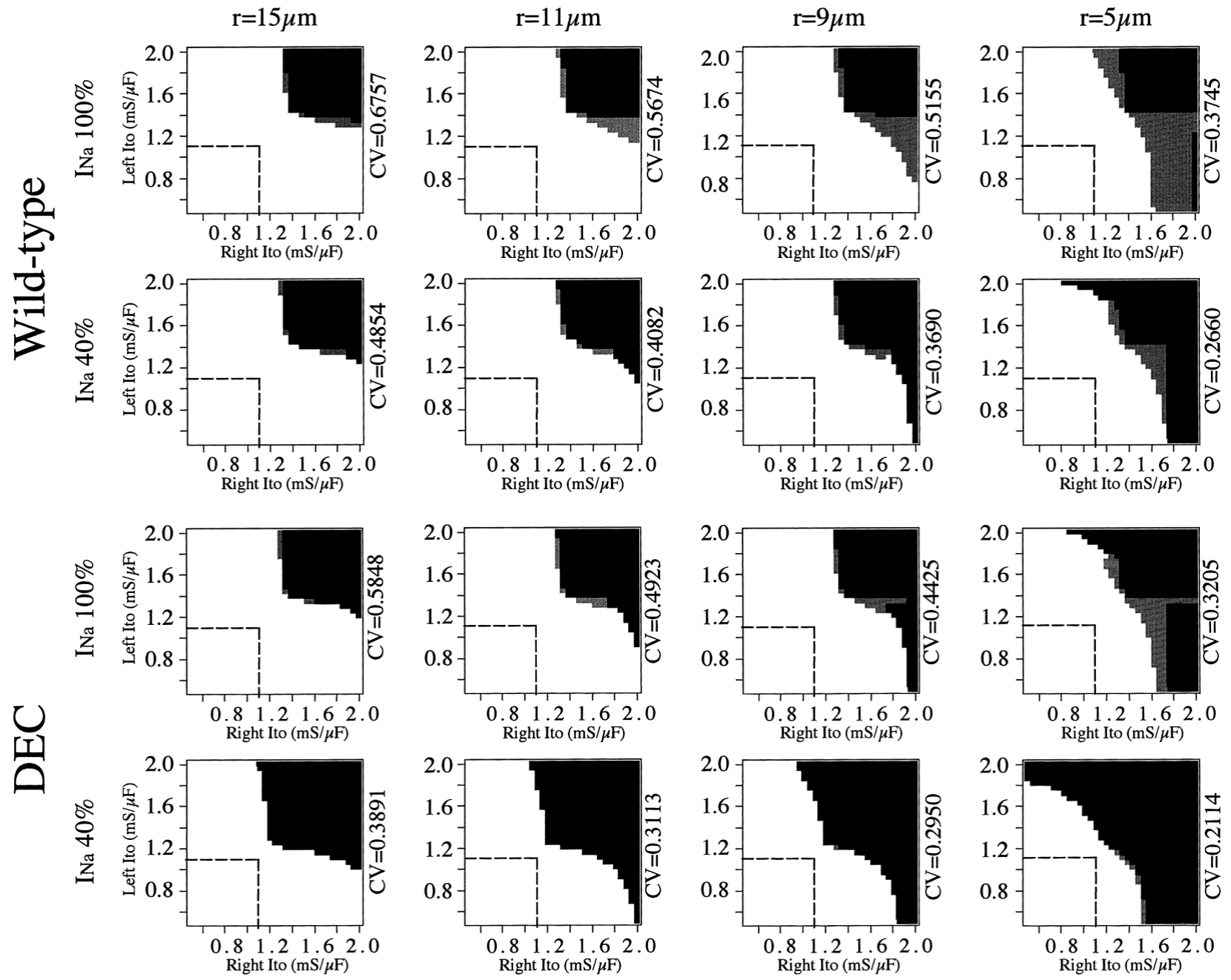

Early repolarization (ER)

Phase 2 reentry (P2R)

\section{Non-ER}

Fig 7. Occurrence of P2R and ER as a result of dispersion at an Ito-density with wild-type INa (upper 2 columns) or DEC-INa (lower 2 columns) at various cell radii ( $r=$ radius of the cell along the short axis). Each matrix is shown in the same manner as in Fig 1. The radius of the cell is shown at the top of each matrix, and the calculated CV is shown at the left of each matrix. Conduction velocity decreased significantly and the area of the P2R and ER increased as a function of smaller cell size. The area of P2R approached the physiological Ito range (within the dotted square) particularly closely when the conditions were $\mathrm{r}=5 \mu \mathrm{m}$, a $40 \%$ decrease in INa, and DEC-INa. CV, conduction velocity.

and $40 \%$ INa density, the area of P2R was located closer to the physiological Ito range in DEC-INa. This suggests that not the decrease in conduction velocity but the rapid inactivation in the kinetics directly increased the likelihood of causing P2R at near physiological Ito density.

\section{Discussion}

As stated, ER does not necessarily cause P2R, and thus it was very important to observe P2R in our model to evaluate the incidence of lethal arrhythmia in BS. The present results show that INa as well as ICaL is important in initiating P2R. The finding that INa blockade decreased the pixel area of P2R is consistent with the fact that INa blockers have often been used empirically to prevent VF clinical$1 y^{3,30,31}$ in patients with BS. However, care should be exercised when prescribing INa channel blockers for patients with the SCN5A mutation of the DEC-INa type, because the area of the P2R approaches the physiological Ito-range, leading to a proarrhythmic effect.

The degree of ST-segment elevation often changes dynamically during the clinical follow-up of BS. As a result, in some populations of patients, the ST-segment often becomes normal when there is no $\mathrm{VF}^{3,32,33}$ One of the cellular bases for this ST-segment change is the change in ICaL-density ${ }^{18}$ On the other hand, INa blockers are commonly used to unmask the latent ST-segment elevation in BS and this has been explained by a greater decrease in INa conductance than decrease under the baseline conditions of BS. However, if that were true, strong INa blockade should cause nonspecific ST-segment elevation, even in normal subjects, and thus there must another underlying mechanism. Our finding that the rapid inactivation in INa may be one of the cellular mechanisms might explain why the INa blocker challenge test can unmask ST-segment elevation in BS but not in normal subjects 3,10 As shown in the present and previous studies, ${ }^{18,34,35}$ a prolonged QT interval is often observed in the right precordial lead of symptomatic BS 
patients. Our simulation suggested that a prolonged QT interval, which represents the occurrence of P2R within the epicardial RV, is an important ECG sign for predicting P2R and subsequent VF. The previous studies of the mutant SCN5A channel6,12 simulated ER and a saddle-back type ST-segment elevation, but did not elucidate how the INa channel blocker induced ST-segment elevation, especially of the coved-type.

The P2R pixel area increased dramatically as a function of fiber length, which suggests that not only the SCN5A mutation but also the area of the epicardium with Ito-dispersion is important for the genesis of P2R. This finding is consistent with the clinical observation of a close correlation between the degree of ST-segment elevation and the likelihood of VF in BS3,32,33 because marked ST-segment elevation may reflect a large amount of epicardial tissue with Ito-dispersion. The conduction disturbance must play an important role in the etiology of P2R. The smaller cell size in the RV myocyte compared with those in the LV is commonly observed in the patch clamp experiment. We speculate that the conduction disturbance might occur more easily in the RV than in the LV because the cross-sectional area along the short axis is smaller in RV myocytes than in LV myocytes,22,23 which would preferentially increase the intracellular resistance along the longitudinal axis in the $\mathrm{RV}$. Because the conduction disturbance simulated by an increase in $\mathrm{Rg}$ had the same effect on the occurrence of ER and P2R (data not shown), we simulated a conduction disturbances in the present study by changing the cell diameter. The smaller cell diameter combined with a conduction disturbance in RV epicardium increased the pixel area of ER and P2R, and in turn increased the likelihood of generating P2R closer to the physiological range of Ito-density, especially in DEC-INa. Increased intracellular resistance may cause the loss of electrical coupling, which may exaggerate dispersion and generate ER and P2R within a limited length of fiber. Regional differences in the size of ventricular myocytes, as well as differences in Itodensity, 46 may play a role in the RV dominant occurrence of the electrophysiological features of BS. It is noteworthy that conduction disturbance was necessary to cause P2R by Ito-dispersion at the physiological range in the simulation with DEC-INa.

The overlap between BS and long QT syndrome-3 has been reported to be attributable to a single SCN5A mutation, $6,15,37,38$ The Markov model of INa of this mutant channel reportedly caused ER in the mathematical model 6 Because P2R was also observed in our model, which was based on the Markov model of INa (our preliminary observation), the selection of the INa model does not seem to have caused any essential changes in our results. The Markov model is a more physiological model than the classic Hodgkin-Huxley model; 39 however, we did not select the Markov model as the INa model for the present study because it is difficult to change the voltage dependent kinetics of INa without changing the amplitude of the simulated current.

\section{Study Limitations}

The incidence of the SCN5A gene mutation in BS is reported as $10-20 \%$. Furthermore only 2 mutations (S1710L ${ }^{16}$ and $\mathrm{T} 1620 \mathrm{M}^{12}$ ) have been reported to show a small inactivation time constant. Therefore, our present study did not show that all BS patients have the DEC-INa mutation, but does show that this current phenotype can easily cause the
BS phenotype. Moreover, it has been reported that a patient with the S1710L mutation did not show Brugada-specific ST-T abnormality ${ }^{16}$ On the other hand, there was not a clear description of the T1620M mutation. However, as the occurrence of the P2R depends on the conductance of ICaL, as shown in the previous study, the sensitivity of the drug challenge test might not be very high. Furthermore, the ECG change in BS is observed only in a localized area ${ }^{40}$ in the right precordial lead. So even if a Brugada-specific ECG change is observed in an electrode, sometimes no change is observed in an adjacent electrode. In particular, the amplitude of the ECG in V3R or V4R is usually low so electrophysiological change in the localized RV free wall might not be observed as a clear Brugada-type ECG change. The other type of change in the current phenotype may contribute to the ECG-phenotype of BS patients who have persistent ST-segment elevation. Finally, although our results from the simulation assist greatly in understanding the mechanism of P2R, they cannot be applied directly to the clinical situation.

\section{Acknowledgment}

This work was supported by the Research Grant for Cardiovascular Disease (13-1) from the Ministry of Health, Labor and Welfare Japan and The Suntory Fund and Advanced Cardiac Therapeutics Keio University School of Medicine.

\section{References}

1. Brugada P, Brugada J. Right bundle block, persistent ST segment elevation and cardiac death: A distinct clinical and electrocardiographic syndrome. J Am Coll Cardiol 1992; 20: 1391-1396.

2. Sumiyoshi M, Nakata Y, Hisaoka T, Ogura S, Nakazato Y, Kawai S, et al. A case of idiopathic ventricular fibrillation with incomplete right bundle branch block and persistent ST segment elevation. Jpn Heart J 1993; 34: 661-666.

3. Miyazaki T, Mitamura H, Miyoshi S, Soejima K, Aizawa Y, Ogawa $\mathrm{S}$. Autonomic and antiarrhythmic drug modulation of ST segment elevation in patients with Brugada syndrome. J Am Coll Cardiol 1996; 27: 1061-1070.

4. DiDiego J, Sun Z, Antzelevitch C. Ito and action potential notch are smaller in left vs right canine ventricular epicardium. Am J Physiol 1996; 271: H548-H561.

5. Yan GX, Antzelevitch C. Cellular basis for the Brugada syndrome and other mechanisms of arrhythmogenesis associated with ST-segment elevation. Circulation 1999; 100: 1660-1666.

6. Gima K, Rudy Y. Ionic current basis of electrocardiographic waveforms: A model study. Circ Res 2002; 90: 889-896.

7. Lukas A, Antzelevitch C. Phase 2 reentry as a mechanism of initiation of circus movement reentry in canine epicardium exposed to simulated ischemia. Cardiovasc Res 1996; 32: 593-603.

8. Krishnan S, Antzelevitch C. Flecainide-induced arrhythmia in canine ventricular epicardium: Phase 2 reentry? Circulation 1993; 87: 562 572.

9. DiDiego JM, Antzelevitch C. Pinacidil-induced electrical heterogeneity and extrasystolic activity in canine ventricular tissues: Does activation of ATP-regulated potassium current promote phase 2 reentry? Circulation 1993; 88: 1177-1189.

10. Brugada J, Brugada P. Further characterization of the syndrome of right bundle branch block, ST segment elevation, and sudden cardiac death. J Cardiovasc Electrophysiol 1997; 8: 325-331.

11. Chen Q, Kirsch GE, Zhang D, Brugada R, Brugada J, Brugada P, et al. Genetic basis and molecular mechanism for idiopathic ventricular fibrillation. Nature 1998; 392: 293-296.

12. Dumaine R, Towbin JA, Brugada P, Vatta M, Nesterenko DV, Nesterenko VV, et al. Ionic mechanisms responsible for the electrocardiographic phenotype of the Brugada syndrome are temperature dependent. Circ Res 1999; 85: 803-809.

13. Bezzina C, Veldkamp MW, van Den Berg MP, Postma AV, Rook $\mathrm{MB}$, Viersma JW, et al. A single $\mathrm{Na}(+)$ channel mutation causing both long-QT and Brugada syndromes. Circ Res 1999; 85: 12061213.

14. Rook MB, Bezzina Alshinawi C, Groenewegen WA, van Gelder IC, van Ginneken AC, Jongsma HJ, et al. Human SCN5A gene muta- 
tions alter cardiac sodium channel kinetics and are associated with the Brugada syndrome. Cardiovasc Res 1999; 44: 507-517.

15. Deschenes I, Baroudi G, Berthet M, Barde I, Chalvidan T, Denjoy I, et al. Electrophysiological characterization of SCN5A mutations causing long QT (E1784K) and Brugada (R1512W and R1432G) syndromes. Cardiovasc Res 2000; 46: 55-65.

16. Akai J, Makita N, Sakurada H, Shirai N, Ueda K, Kitabatake A, et al. A novel SCN5A mutation associated with idiopathic ventricular fibrillation without typical ECG findings of Brugada syndrome. FEBS Lett 2000; 479: 29-34.

17. Clancy $\mathrm{CE}$, Rudy $\mathrm{Y}$. $\mathrm{Na}(+)$ channel mutation that causes both Brugada and long-QT syndrome phenotypes: A simulation study of mechanism. Circulation 2002; 105: 1208-1213.

18. Miyoshi S, Mitamura H, Fujikura K, Fukuda Y, Tanimoto K, Hagiwara Y, et al. A mathematical model of phase 2 reentry: Role of L-type Ca current. Am J Physiol 2003; 284: H1285-H1294.

19. Fujiki A, Usui M, Nagasawa H, Mizumaki K, Hayashi H, Inoue H. ST segment elevation in the right precordial leads induced with class IC antiarrhythmic drugs: Insight into the mechanism of Brugada syndrome. J Cardiovasc Electrophysiol 1999; 10: 214-218.

20. Ikeda T, Sakurada H, Sakabe K, Sakata T, Takami M, Tezuka N, et al. Assessment of noninvasive markers in identifying patients at risk in the Brugada syndrome: Insight into risk stratification. $J$ Am Coll Cardiol 2001; 37: 1628-1634.

21. Aizawa Y, Tamura M, Chinushi M, Naitoh N, Uchiyama H, Kusano $\mathrm{Y}$, et al. Idiopathic ventricular fibrillation and bradycardia-dependent intraventricular block. Am Heart J 1993; 126: 1473-1474.

22. Gerdes AM, Moore JA, Hines JM, Kirkland PA, Bishop SP Regional differences in myocyte size in normal rat heart. Anat Rec 1986; 215: 420-426.

23. Smolich JJ, Walker AM, Campbell GR, Adamson TM. Left and right ventricular myocardial morphometry in fetal, neonatal, and adult sheep. Am J Physiol (Heart Circ Physiol) 1989; 257: H1 - H9.

24. Luo CH, Rudy Y. A dynamic model of the cardiac ventricular action potential: I: Simulations of ionic currents and concentration changes. Circ Res 1994; 74: 1071-1096.

25. Shaw RM, Rudy Y. Ionic mechanisms of propagation in cardiac tissue. Roles of the sodium and L-type calcium currents during reduced excitability and decreased gap junction coupling. Circ Res 1997; 81: 727-741

26. Crank J, Nicolson P. A practical method for numerical evaluation of solutions of partial differential equations of the heat conduction type. Proc Cambridge Phil Soc 1947; 43: 50-67.

27. Antzelevitch C, Jalife J, Moe GK. Characteristics of reflection as a mechanism of reentrant arrhythmias and its relationship to parasystole. Circulation 1980; 61: 182-191.
28. Jalife J, Antzelevitch C, Moe GK. The case for modulated parasystole. Pacing Clin Electrophysiol 1982; 5: 911 -926.

29. Plonsey R. The active fiber in a volume conductor. IEEE Trans Biomed Eng 1974; 21: 371-381.

30. Chinushi M, Aizawa Y, Ogawa Y, Shiba M, Takahashi K. Discrepant drug action of disopyramide on ECG abnormalities and induction of ventricular arrhythmias in a patient with Brugada syndrome. $J$ Electrocardiol 1997; 30: 133-136.

31. Nakazato Y, Nakata Y, Yasuda M, Yamagami S, Tokano T, Nakazato $\mathrm{K}$, et al. Idiopathic ventricular fibrillation initiated by a short-coupled ventricular premature beat. Jpn Heart J 1996; 37: 265-269.

32. Atarashi H, Ogawa S, Harumi K, Hayakawa H, Sugimoto T, Okada $\mathrm{R}$, et al. Characteristics of patients with right bundle branch block and ST-segment elevation in right precordial leads: Idiopathic Ventricular Fibrillation Investigators. Am J Cardiol 1996; 78: 581 583.

33. Kasanuki H, Ohnishi S, Ohtuka M, Matsuda N, Nirei T, Isogai R, et al. Idiopathic ventricular fibrillation induced with vagal activity in patients without obvious heart disease. Circulation 1997; 95: 2277 2285.

34. Krishnan SC, Josephson ME. ST segment elevation induced by class IC antiarrhythmic agents: Underlying electrophysiologic mechanisms and insights into drug-induced proarrhythmia. J Cardiovasc Electrophysiol 1998; 9: 1167-1172.

35. Pitzalis MV, Anaclerio M, Iacoviello M, Forleo C, Guida P, Troccoli $\mathrm{R}$, et al. QT-interval prolongation in right precordial leads: An additional electrocardiographic hallmark of Brugada syndrome. J Am Coll Cardiol 2003; 42: $1632-1637$.

36. Litovsky SH, Antzelevitch C. Transient outward current prominent in canine ventricular epicardium but not endocardium. Circ Res 1988; 62: 116-126.

37. Baroudi G, Chahine M. Biophysical phenotypes of SCN5A mutations causing long QT and Brugada syndromes. FEBS Lett 2000; 487: $224-228$

38. Priori SG, Napolitano C, Schwartz PJ, Bloise R, Crotti L, Ronchetti E. The elusive link between LQT3 and Brugada syndrome: The role of flecainide challenge. Circulation 2000; 102: 945-947.

39. Hodgkin AL, Huxley AF. A quantitative description of membrane current and its application to conduction and excitation in nerve. $J$ Physiol (London) 1952; 117: 500-544.

40. Hisamatsu K, Morita H, Kusano FK, Takenaka S, Nagase S, Nakamura K, et al. Evaluation of usefulness of recording the ECG in the 3rd intercostal space and prevalence of Brugada-type ECG in accordance with recently established electrocardiographic criteria. Circ J 2004; 68: 135-138. 